\title{
MANEJO QUIRÚRGICO DE LAS FRACTURAS ORBITARIAS
}

\section{Hospital de San José. 1997-2002*}

Soraya Salej Higgins MD. ${ }^{* *}$, René Pedraza Alarcón MD. ${ }^{* * *}$, Martín Pinzón Navarro MD. ${ }^{* * *}$

\section{Resumen}

Se revisaron de modo retrospectivo 25 pacientes con fracturas orbitarias, intervenidos quirúrgicamente en el Hospital de San José, por indicación funcional y/o estética, entre enero de 1997 y marzo de 2002. Los accidentes automovilísticos, junto con la agresión, fueron los mecanismos más comunes $(84 \%)$. El sexo masculino fue el más afectado (84\%). Para la clasificación de las fracturas se empleó el sistema de puras e impuras (Converse y Smith), con predominio de las últimas (88\%). La vía de abordaje fue combinada en el $90 \%$ de los procedimientos, prefiriéndose para el piso de la órbita la subciliar y transconjuntival, y los abordajes clásicos para el techo y la pared lateral. Para la reconstrucción orbitaria se emplearon: malla de titanio en 13 pacientes, injertos autólogos (septum, calota, cresta ilíaca y fascia temporal) en 11 individuos, e injerto de banco de huesos en uno. La presencia de secuelas se relacionó con el trauma y el método de reconstrucción, así como con el abordaje empleado y el tiempo transcurrido al momento de la intervención. El $48 \%$ de los casos presentó secuelas: muestra escleral en seis pacientes, seguida por enoftalmos y diplopía en tres. Sólo uno de ellos (4\%) ameritó reintervención. La muestra escleral se asoció en cinco sujetos con abordaje subciliar. Ninguno de los pacientes presentó infección o rechazo del injerto. Todos los individuos con distopia ocular en el postoperatorio, fueron sometidos a reconstrucción con injertos autólogos.

\section{Introducción}

El esqueleto orbitario, conformado por siete huesos faciales, es una encrucijada anatómica importante debido a su relación estrecha con el sistema nervioso central, nariz, senos paranasales, cara y estructuras relacionadas con el soporte y la función orbitaria.

La historia de la cirugía maxilofacial registra controversias sobre el manejo de las fracturas orbitarias, en cuanto a la decisión de operar o no, realizar el procedimiento temprano o tardío, cuál es la mejor forma de evaluar el paciente y cómo definir la técnica ideal para restaurar la función y la apariencia.

Trabajo presentado en el XXXI Congreso de la Sociedad Colombiana de ORL y Cirugia Maxilofacial en el mes de mayo del año 2002 en la ciudad de Santa Marta.

- Fellow de Cirugia Oral y Maxilofacial. Hospital de San José.

*.. Jefe del Servicio de Cirugia Oral y Maxilofacial. Hospital de San josé.

*. Instructor servicio ORL - Cirugia Oral y Maxilofacial. Hospital de San José.

Correspondencia a:

Soraya Sale

Diag 127 A \# 29- 88. Horizonte Health Resources. Cons 422

E-mail: sorayahi@hotmail.com
El Hospital de San José, como pionero en el tratamiento del trauma facial en Colombia, tiene enorme experiencia en el manejo de las fracturas orbitarias. Por ello se recopiló en este trabajo descriptivo los casos manejados mediante cirugía en los últimos cinco años, el comportamiento epidemiológico de esta patología, su manejo y las secuelas principales.

\section{Marco teórico}

La órbita es la única estructura ósea que tiene como propósito primario albergar y proteger el globo ocular. Es una pirámide de cuatro lados con el ápice en el foramen óptico y una base anterior que corresponde al reborde orbitario. Está conformada por los huesos maxilar, zigomático, frontal, etmoides, lacrimal, palatino y esfenoides. Las paredes orbitarias son variables en cuanto a grosor y fortaleza.

Las fracturas de los dos tercios anteriores son las más comunes, pero allí los senos etmoidales y maxilares absorben la energía del impacto, por lo cual las le- 
siones internas del globo son poco frecuentes. Por lo regular están acompañadas por fracturas del tercio facial medio y van desde las conocidas como blow out hasta las que producen disrupción de la órbita.

Sehan hecho muchos intentos porclasificarlas; Smith y Regan en $1957^{\prime}$ describieron por primera vez el término blow out, para referirse a las fracturas puras de órbita que no comprometen el reborde orbitario. Por su parte, Jackson ${ }^{2}$ las clasifica en zigomático-orbitarias, naso-órbito-etmoidales e internas (lineales, blow out y complejas). Sin embargo, para fines prácticos, la clasificación utilizada en el servicio del Hospital de San José divide las fracturas orbitarias en puras (blow out y blow in) e impuras (según la clasificación deConverse y Smith de 1960). ${ }^{3}$

Se define como fractura blow out aquella que compromete cualquier pared de la órbita sin compromiso del reborde. En orden de prevalencia se afectan las paredes inferior, medial, lateral y superior.' Se produce también por rotación de los fragmentos óseos de la porción central de la fractura denominándose en dicho caso fracturas "trap door". Si el trauma involucra uno de los rebordes se considera impura, situación que por lo general se encuentra en las fracturas malares, maxilares, naso-orbito-etmoidales y frontales.

El defectocreado por las fracturas es variable y puede llenarse con tejidos blandos de la órbita produciendo la apariencia de hipoftalmos y/o enoftalmos. La grasa herniada contiene el septum fibroso, lo cual altera la actividad mecánica de los músculos extraoculares. Los cambiosen la posición del globo pueden tener impacto en la tensión y contracción de dichos músculos. Además, existe la posibilidad de lesión directa sobre estructuras nerviosas y musculares.

Cualquiera de los factores mencionados puede alterar la mirada conjugada y producir diplopía. ${ }^{1-4}$ Por lo anterior, debe determinarse en el examen clínico el mecanismo y dirección del trauma, siendo además obligatoria una valoración por oftalmología, puesto que 0,6 a $4 \%$ de los pacientes con fractura orbitaria sufren pérdida significativa o completa de la visión. ${ }^{24}$ Los movimientos oculares se determinan para buscar la presencia de atrapamiento ocular o paresia. Es importante registrar la presencia de diplopía, pero si aparece sólo en posición extrema de la mirada puede ser debida al edema. Siempre se debe realizar una prueba de ducción forzada para determinar si la paresia es por atrapamiento ocular o por lesión neuromuscular.

Las técnicas de imagen son esenciales en el diagnóstico y manejo terapéutico, en especial la tomografía axial computarizada (TAC), que constituye la prueba radiológica estándar para las fracturas orbitarias, pues permite una buena valoración del marco esquelético, de los tejidos blandos y del cerebro. Para ello se necesitan imágenes en dos planos, coronal y axial, con cortes de $3 \mathrm{~mm}$. La resonancia magnética (RM) tiene una utilidad limitada en el trauma orbitario, pero es utilizada para valorar las lesiones intraoculares y de tejidos blandos no muy bien definidas con la TAC y la detección de cuerpos extraños metálicos. ${ }^{2}$

Existen indicaciones absolutas y relativas de exploración y cirugía correctiva para evitar posibles secuelas. ${ }^{2.5 .6}$ Entre las absolutas tenemos enoftalmos agudo (de más de 2 ó $3 \mathrm{~mm}$ ) e hipoftalmos (mayor de 2 a $3 \mathrm{~mm}$ ), así como la restricción mecánica de la mirada asociada a diplopía en la prueba de ducción forzada y evidencia radiológica de atrapamiento muscular. Entre las indicaciones relativas encontramos en primer lugar la presencia de un área de 1,5 a $2 \mathrm{~cm}$ de pared desplazada o un defecto mayor del $50 \%$ del piso o pared medial, detectados mediante TAC. En segundo lugar la persistencia de diplopía con una prueba de ducción forzada negativa, sin evidencia de compromiso nervioso.

Por lo anterior, algunos autores proponen que ante la duda entre atrapamiento muscular y de la periórbita, es conveniente dar un margen de espera de siete a diez días y manejo con corticoides sistémicos. Si no hay mejoría significativa (con diplopía en mirada a 30 grados de la posición de referencia) se debe realizar exploración quirúrgica. Las disrupciones que comprometen menos de un $50 \%$ del suelo orbitario sin deformidad estética clara, precisan seguimiento clínico durante dos semanas. Si durante este período aparece enoftalmos mayor de $2 \mathrm{~mm}$ ohipoftalmos, se procede a manejo quirúrgico. 
Los abordajes quirúrgicos más usados para el reborde orbitario inferior son el subciliar, transconjuntival e infraorbitario. ${ }^{1-5}$ Para la región supero lateral se utilizan cola de ceja, blefaroplastia modificada, bicoronal o cantotomía lateral. Para la pared medial son de utilidad el bicoronal o abordaje de Lynch, en especial para reconstruir el ligamento cantal medial avulsionado, que ocurre por lo regular en fracturas naso-órbito-etmoidales (NOE) y tipo Le Fort 111.

Después de exponer las fracturas, se realiza la reducción de los segmentos, comenzando casi siempre por las áreas estables como el hueso firontal, arco zigomático o mandíbula. Cuando la fractura orbitaria coexiste con otras faciales, éstas deben reducirse primero. Se debe restablecer la altura, ancho y proyección del tercio medio facial.

Previo al advenimiento de la era de los dispositivos de fijación interna rígida, se emplearon elementos de fijación externa. Los alambres de acero inoxidable se usaron a menudo antes de la introducción de placas y tornillos. Los alambres interóseos producen compresión de los fragmentos, pero no hay estabilidad torsional o rotacional, además de la difïcultad que ofrecen para actuar de manera diferente con los fragmentos pequeños. Entre las complicaciones relacionadas con los alambres están la infección, migración, hipersensibilidad y perforación del globo ocular.?

La fijación interna rígida con placas y tornillos demuestra ser superior a los alambres en el reparo de las fracturas del tercio medio. ${ }^{7-11}$ Su uso es más bien nuevo en el continente americano, pero en Europa se emplea desde hace 4 décadas. Se utilizan miniplacas con tomillos de 1,3 a 2 mm de diámetro en los pilares mediales y laterales del tercio medio facial. El sistema de microplacas emplea tornillos de 1 mm o menos de diámetro. en tercio superior facial, en especial en el reborde orbitario y lazona firontal.

Grandes fiacturas que comprometen el piso orbitario y pared medial pueden reconstruirse con material aloplástico o injertos óseos. Con el uso de mallas de titanio se logra buena corrección del enoftalmos mediante la restauración del contorno y el volumen. Entre las ventajas que ofrece está la reconstrucción de la for- ma del tercio posterior de la órbita, mejor que con injertos óseos.

\section{Materiales y métodos}

El presente estudiodescriptivo observacional histórico tipo serie de casos, incluye 2.5 pacientes intervenidos por fracturas orbitarias entre enero de 1997 y marzo de 2()()2 en el servicio de ORL y Cirugía Oral y Maxilofacial del Hospital de San José.

Se diseñó una base de datos con variables preoperatorias como edad, sexo, tiempo transcurrido al momento de la cirugía, mecanismo del trauma, signos clínicos prequirúrgicos, hallazgos en TAC y tipo de fractura, así como variables intraperatorias como abordajes, material utilizado y técnica, al igual que secuelas post operatorias.

El tratamiento estadóstico de la información se realizó con el programa SPSS versión 10,(). Se describe la firecuencia (proporción) de cada variable en el grupo de estudio. Para el tiempo transcurrido al momento de la cirugía, la descripción de la distribución, se hizo utilizando la mediana.

La clasificación utilizada fue la de Converse y Smith:

\section{- PURAS}

- Blow out

- Blow in

\section{- IMPURAS}

- Órbito-zigomáticas

- Naso-órbito-etmoidales (NOE)

- Seno frontal

- Le Fort II y III

\section{Resultados}

La edad media fue de 33 años más o menos 14,2 (rango de 10 a 75 años), con mayor proporción en la segunda a cuarta década de la vida. Predominio del sexo masculino con $84 \%$ y una mediana para el tiempo de evolución de la fractura al momento de la intervención de 29 días (rango entre 5 y 180 días). Del total, 21 de los pacientes fucron intervenidos en el primer mes y cuatto después de 2 meses post fractura, debido al estado general y las lesiones sistémicàs asociadas. 
Los accidentes de tránsito y la agresión fueron los mecanismos más frecuentes de trauma con $60 \%$ y $24 \%$, respectivamente. En menor proporción estuvieron los accidentes deportivos, caídas y heridas por arma de tuego.

Las indicaciones quirúrgicas de reconstrucción orbitaria fueron de tipo funcional y/o estético, siendo el enoftalmos la más firecuente en 14 pacientes, la diplopía en 10 y la proptosis en uno (Figura 1 ).

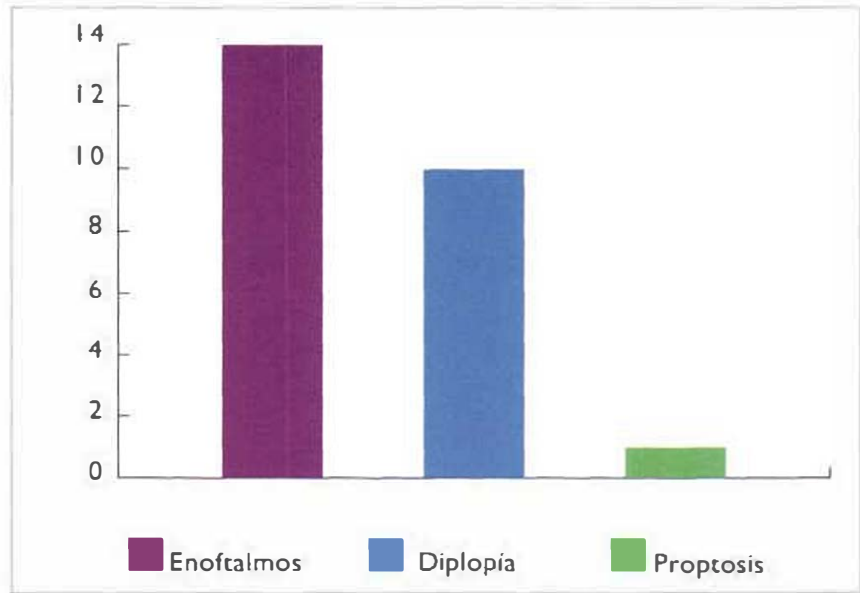

Figura I. Fracturas orbitarias según hallazgos.

Los hallazgos clínicos más fiecuentes fueron la equimosis periorbitaria en 24 de los pacientes (excepto en uno que fue manejado como secuela a los seis meses). la presencia de escalón en 20) y parestesia infraorbitaria en 17. El 64\% de los casos presentaron alteración de la agudeza visual ( Figura 2 ).

La Figura 3 muestra la distribución según la clasiticación de Wyte. Nótese que fueron más frecuentes las fracturas impuras, en primer lugar la órbito-zigomática con $60 \%$ ( 15 pacientes), seguido por las NOE y del seno frontal con $32 \%$ y $24 \%$, respectivamente. Las menos comunes fueron las fracturas puras con tres individuos.

Al $10(0 \%$ de los sujetos se les practicó TAC obteniéndose información de las paredes orbitarias afectadas: piso orbitario (20) pacientes), medial (13), lateral (12) y techo orbitario (7).

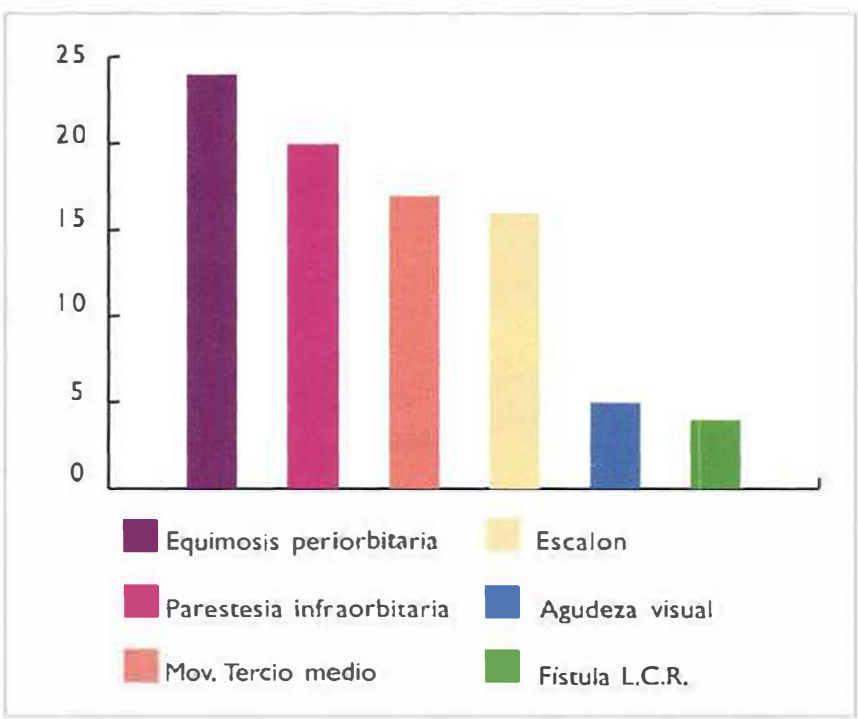

Figura 2. Fracturas orbitarias según hallazgos.

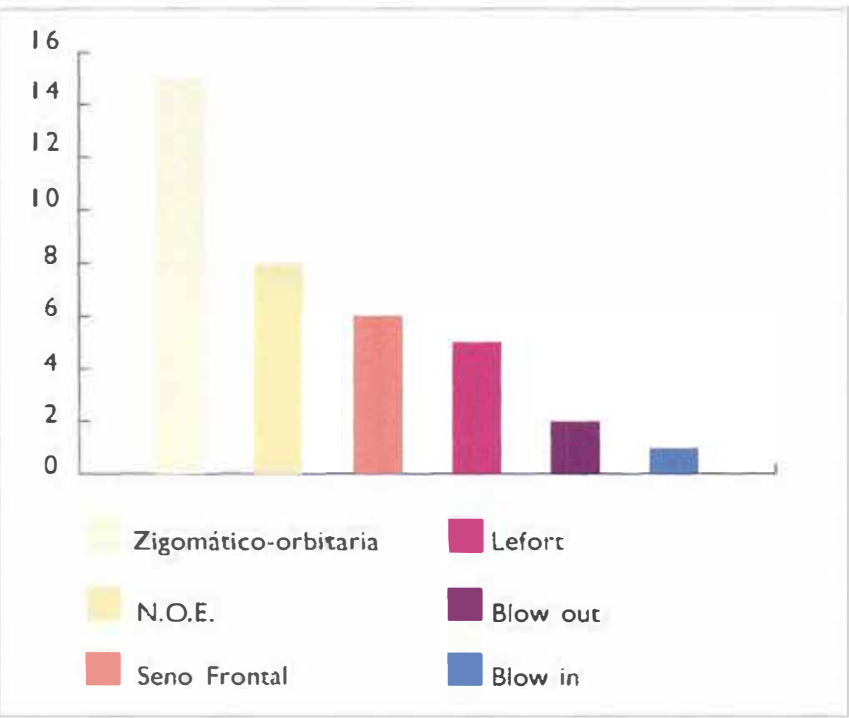

Figura 3. Fracturas orbitarias según clasificación.

El 68\% de los pacientes presentaron compromiso ocular, locual coincide con la literatura mundial, siendo la más fiecuente la neuropatía óptica post- traumática. Uno de los pacientes desarrolló ceguera secundaria a hematoma retro-ocular ( Figura 4).

En quienes tuvieron limitación de los movimientos oculares, el músculo más atrapado fue el recto inferior en cinco pacientes, seguido del recto lateral en tres y del recto superior en uno. 


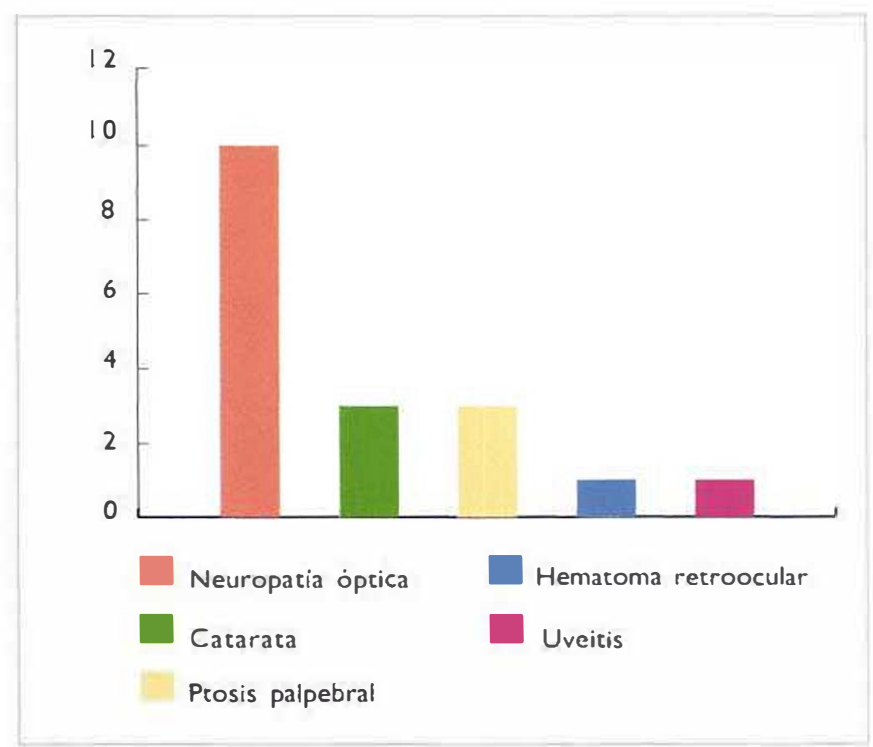

Figura 4. Fracturas orbitarias según compromiso ocular.

Los abordajes para la reducción anatómica fueron combinados en el 90\% de los casos, distribuidos así: subciliar en 15 pacientes, yugal en 14 y transconjuntival en seis, así como blefaroplastia modificada y cola de ceja en cinco casos cada uno. En fracturas complejas de terciosuperior se prefirió el abordaje coronal, combinado en ciertasocasionescon subciliar y/o yugal.

Durante la reconstrucción del contorno orbitario se empleó malla de titanio en 13, injerto autólogo en 11 y en uno en uno injerto de banco de huesos. (Figura 5). De los injertos autólogos se prefirió el septum en cuatro pacientes y cresta ilíaca en tres, seguido por la calota en

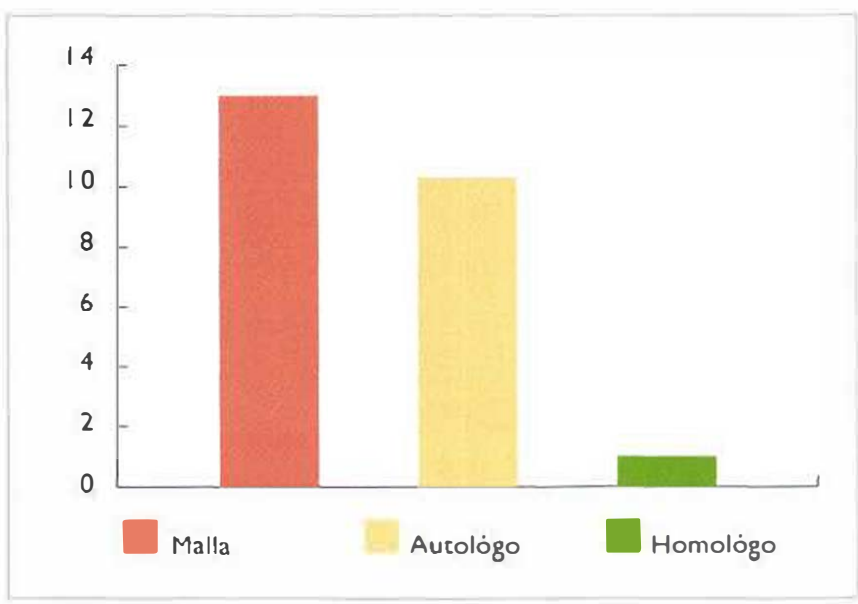

Figura 5. Fracturas orbitarias según manejo. sólo dos. En dos de los enfermos se combinó la utilizalción de malla con fascia de músculo temporal.

La presencia de secuelas, que ocurrió en $48 \%$ de los sujetos. se relacionó con el trauma en sí, con el método de reconstrucción utilizado y con el abordaje empleado. La más frecuente fue la muestra escleral en 6 pacientes, seguido por enoftalmos y diplopía, con 3 casos cada uno. Sólo uno de estos pacientes (4\%) ameritó reintervención, debido a que dichos hallazgos fueron por lo general transitorios y hubo remisión de los signos en menos de 3 semanas. El paciente que presentó queloide en el postoperatorio se le practicó abordaje cola de ceja (Figura 6).

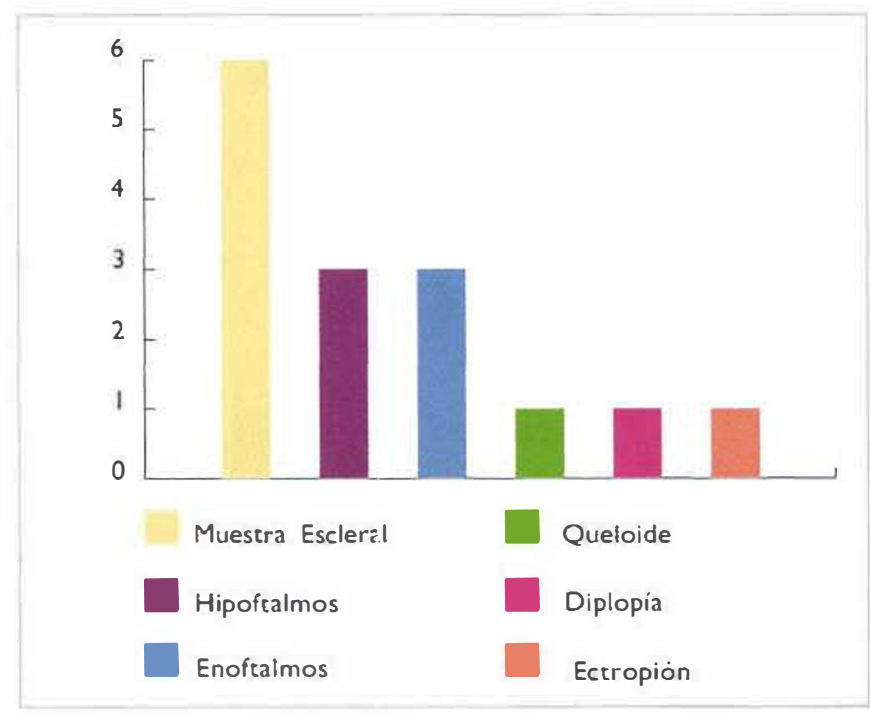

Figura 6. Fracturas orbitarias según secuela.

\section{Conclusiones;}

Los hallazgos del presente trabajo comparados con la literatura internacional no presentan grandes variaciones. El sexo más afectado es el masculino y el rango de edad va de la segunda a la cuarta década de la vida. Con el advenimiento de los accidentes de alta velocidad se incrementó la incidencia a nivel mundial de las fracturas faciales. Los accidentes de tránsito y la agresión fueron las principales causas de traumatismocon un $84 \%$ de la totalidad. Los accidentes de mayor impacto y entre ellos las fracturas Le Fort II, se asociaron a un mayor compromiso de la agudeza visual. 
La presencia de secuelas se relacionó con el trauma y con el método de reconstrucción utilizado, así como con el abordaje empleado y el tiempo transcurrido al momento de la intervención. En cinco de seis pacientes en los cuales se empleó el abordaje subciliar para el piso orbitario, se evidenció muestra escleral en postoperatorio. Los dos pacientes intervenidos tardíamente presentaron enoftalmos post-quirúrgico. Uno de ellos requirió reintervención por secuelas funcionales y estéticas. Ninguno desarrolló infección o rechazo del injerto. La totalidad de los enfermos con distopia ocular en el postoperatorio fueron reconstruidos con injertos autólogos.

\section{Bibliografía}

1. Burm J, Cheng CH. Pure orbital blowout fractures: new concepts and importance of medial orbital blow out fracture. Plastic Reconstr Surg 1999; 103: 1839-49
2. Gonty A, Fonseca R. Management of frontal sinus fractures and associated injuries. Oral and Maxilofacial Surgery, W.B. Saunders 2000;3:301-326

3. Gonty A, Fonseca R, Orbital trauma, Oral and Maxilofacial Surgery W.B. Saunders 2000; 3: 205-299

4. Schendel S, Orbital trauma. Oral and Maxilofacial Surgery Clinics of North America 1993;5(3)

5. Harstein M, Update on orbital floor fractures: Indications and timing for repair. Facial Plastic Surgery 2000; 16:95-106

6. Bowerman J, Williams J, Fractures of the middle third of the facial skeleton. Maxilofacial Injuries. Churchill Livingstone, 1994; 2: 591-663

7. Hoffmann J. NOE complex fractures management. Facial Plastic Surgery 1998; 14:67-76

8. Buphendra CK, Patel B. Management of complex orbital fractures. Facial plastic Surgery 1998; 14: 83-104

9. Shumrick K. Management of the orbital rim and floor in zigoma and midface fractures: criteria for selective exploration. Facial Plastic Surgery 1998; 14: 77-81

10. Courtney DJ, Isolated orbital blowout fractures: Survey and review. Br J Oral Maxilofacial Surg 2000; 38: 496-504

\section{FUNDACIÓN UNIVERSITARIA DE CIENCIAS DE LA SALUD HOSPITAL DE SAN JOSE}

Personería Jurídica No. 10917 del 01-12-1976 Resolución Ministerio de Educación Nacional No, 0125

Facultad de Instrumentación Quirúrgica

Código ICFES 270246100281100111100

Título otorgado

\section{Instrumentador Quirúrgico}

Oficina de admisiones: Calle 10 No. 18-75 Edificio docente, $1^{\text {er }}$ piso PBX. 5998977 • 5998842 TELEFAX $2019867 \cdot 2018938$

E-mail: instrumentacion@fucsalud.edu.co

hppt: www.fucsalud.edu.co

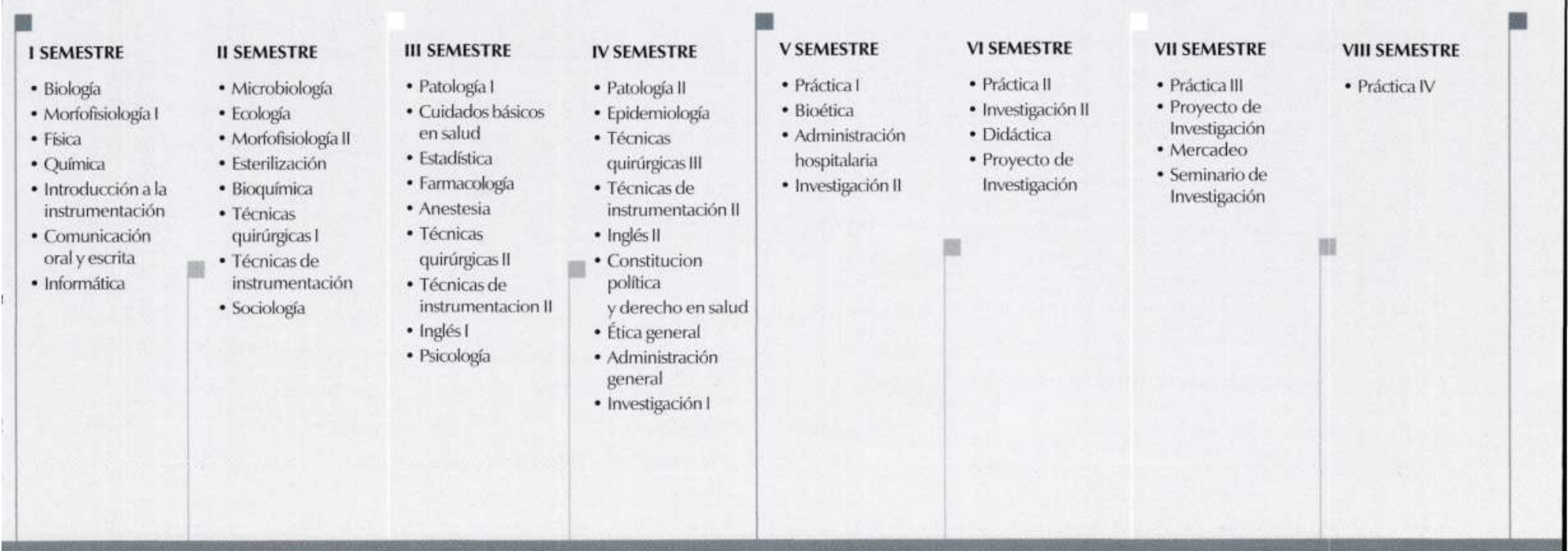

